\title{
A dual perspective on first-session therapeutic alliance: strong predictor of youth mental health and addiction treatment outcome
}

\author{
Patty van Benthem ${ }^{1,2} \cdot$ Renske Spijkerman ${ }^{1} \cdot$ Peter Blanken $^{1} \cdot$ Marloes Kleinjan $^{3,4} \cdot$ Robert R. J. M. Vermeiren $^{5,2}$. \\ Vincent $M$. Hendriks ${ }^{1,2}$
}

Received: 9 July 2019 / Accepted: 25 February 2020 / Published online: 10 March 2020

(c) The Author(s) 2020

\begin{abstract}
We investigated the potential role of first-session therapeutic alliance ratings to serve as an early marker of treatment outcome in youth mental health and addiction treatment. The present study is among the first to incorporate both a youths' and a therapists' perspective of the therapeutic alliance in order to maximize predictive value of the alliance for treatment outcome. One hundred and twenty-seven adolescents participated in a multi-site prospective naturalistic clinical cohort study, with assessments at baseline and at 4 months post-baseline. Main outcome measure was favorable or unfavorable treatment outcome status at 4-month follow-up. Early therapeutic alliance had a medium and robust association with treatment outcome for youth' $(b=1.29)$ and therapist' $(b=1.12)$ perspectives and treatment setting. Based on the two alliance perspectives four subgroups were distinguished. Incorporating the alliance-ratings from both perspectives provided a stronger predictor of treatment outcome than using one perspective. Youth with a strong alliance according to both perspectives had an eightfold odds of favorable treatment outcome compared with youth with a weak alliance according to both perspectives. The association between therapeutic alliance and treatment outcome in youth mental health and addiction treatment may be substantially stronger than earlier assumed when both a youths' and therapists' perspective on alliance is considered.
\end{abstract}

Keywords Therapeutic alliance $\cdot$ Youth $\cdot$ Substance use disorder $\cdot$ Mental health $\cdot$ Treatment outcome

\section{Introduction}

Presentation information: Poster, 16th conference of the European Association for Research on Adolescence, Ghent, Belgium, September 13-15, 2018; oral presentation, 4th International Congress of Clinical and Health Psychology on Children and Adolescents, Palma de Mallorca, Spain, November 15-17, 2018; oral presentation at XVIII Congress of the European Society for Child and Adolescent Psychiatry, Vienna, Austria, 30 June-2 July 2019.

Patty van Benthem

p.vanbenthem@brijder.nl

Renske Spijkerman

Renske.spijkerman@brijder.nl

Peter Blanken

Peter.Blanken@brijder.nl

Marloes Kleinjan

Kleinjan@trimbos.nl

Robert R. J. M. Vermeiren

r.r.j.m.vermeiren@curium.nl

Vincent M. Hendriks

Vincent.Hendriks@brijder.nl
Mental disorders with severe impairment-including substance use disorders (SUD) - affect about one in four to five youths aged 13-18 years in their lifetime [1, 2]. For these young people a range of evidence-based treatments is available, including cognitive behavioral therapy and family-based treatments. However, not all youth who receive

1 Parnassia Addiction Research Center (PARC), Brijder Addiction Care, Zoutkeetsingel 40, 2512 HN The Hague, The Netherlands

2 Department of Child and Adolescent Psychiatry, Curium-LUMC, Leiden University Medical Center, Leiden, The Netherlands

3 Epidemiology and Research Support, Trimbos Institute: Netherlands Institute of Mental Health and Addiction, Utrecht, The Netherlands

4 Department of Interdisciplinary Social Science, Youth Studies, Utrecht University, Utrecht, The Netherlands

5 Youz, Parnassia Group, The Hague, The Netherlands 
mental health treatment benefit from treatment. Weisz et al. [3] found a mean between-groups effect-size of Cohen's $d=0.46$ (medium effect) at the end of treatment, in a metaanalysis of 447 randomized controlled trials of psychological youth mental health therapies. Treatment effect-sizes differed substantially across the targeted disorders, but remarkably were not moderated by type of treatment offered in the experimental conditions of the trials, although most robust evidence was found for behavioral treatments. With one exception, no studies of youth with SUD were included in Weisz et al. [3]. However, meta-analyses of youth with SUD showed comparable treatment effectiveness across different treatment types $[4,5]$. Thus, a considerable proportion of treatment-seeking adolescents do not benefit from mental health/addiction treatment, no treatment is clearly superior, and it is largely unknown which adolescents benefit most from which type(s) of treatment.

This lack of clear differential effectiveness between theoretically quite diverging treatment approaches has led researchers in the mental health field to broaden their focus to common ("non-specific") factors that are shared by most psychotherapies. From these, the therapeutic alliance-also referred to as working alliance or helping alliance; both terms reflecting the collaborative aspects of the therapistpatient relationship [6] - association with treatment outcome has probably received most attention.

Concerning the association between therapeutic alliance and clinical outcomes of mental health treatment, three meta-analyses were recently published: one on adults [6] and two on youths [7, 8]. In their meta-analysis of 295 studies of psychotherapy among adults, Flückiger et al. [6] found an overall alliance-outcome effect-size of $r=0.28$ (medium effect), with similar effect-sizes across treatment approaches and across alliance perspectives (e.g., therapist or patient). Notably, the effect-sizes of the alliance-treatment outcome association differed across disorders, with highest values for personality disorders and lowest values for substance use and eating disorders.

With regard to youth, Murphy and Hutton [8] found a pooled alliance-outcome effect-size of $r=0.29$ (medium effect) in their meta-analysis of 27 youth psychotherapy studies, and Karver et al. [7] found in their meta-analysis of 28 studies an association of $r=0.19$ (small to medium effect). Effect moderators of the alliance-outcome association in youth were investigated only by Karver et al. [7] and appeared to be comparable with those found by Flückiger et al. [6] in adults: type of treatment (behavioral vs. nonbehavioral) and alliance perspective did not moderate the alliance-outcome association, whereas type of disorder did moderate this association, with particularly low effect-sizes for substance use disorders $(r=0.01)$ and eating disorders $(r=0.05)$. Hence, both the strength of the alliance-outcome association and its clinical moderator- type of targeted disorder-found among youth appears to be similar to that found among adults [6-8].

When measuring therapeutic alliance, time of alliance assessment is an important moderator, with-not surprisingly - weaker associations with outcome when alliance is measured early in the therapeutic process [6]. Nevertheless, measuring alliance as early as possible is clinically relevant to allow its use as an early warning sign of potential patient dissatisfaction, premature treatment termination, and/or unfavorable treatment outcome. In addition, various authors have recommended that, when measuring therapeutic alliance, multiple informants should be involved, because different informants are likely to have different views on the quality of the therapeutic relationship [9, 10]. Furthermore, it has been recommended that the alliance evaluations from these multiple sources should be incorporated to gain more insight into the alliance-treatment outcome association [11]. From the 34 independent studies among youth that were included either in the meta-analyses of Karver et al. [7] and/ or Murphy \& Hutton [8], only 13 studies involved both youth and therapist as informants of early alliance and agreement between both informants was generally low [12-17] or nonsignificant $[18,19]$. Remarkably, the alliance ratings of both sources were not used in any of these studies to investigate their combined predictive value for treatment outcome. In the present study, we aim to fill this gap and (1) investigate the prognostic importance of first-session therapeutic alliance, as perceived by youth and therapist, for outcome of youth mental health and addiction treatment, and (2) examine whether incorporating first-session evaluations from the perspective of both youth and therapist provides more value for predicting treatment outcome than using the evaluations of one perspective.

\section{Method}

\section{Design}

This study was part of the Professional Alliance with Clients in Treatment (PACT) study-a multi-site prospective naturalistic clinical cohort study among adolescents in outpatient youth mental health care (YMHC) and youth addiction care (YAC). For the present study, we used data collected at the first treatment session and at four months post-baseline. We assessed therapeutic alliance as early as possible-i.e. at the end of the first treatment-session-to minimize possible confound due to the effect of early symptom improvement on the perceived therapeutic alliance. We assessed treatment-outcome four months post-baseline because most symptom improvement occurs in these first months of treatment [20-22]. This study was funded by The Netherlands Organization for Health Research and Development (no. 
729101014) and approved by the Medical Ethical Board of the University Medical Center Leiden (P.15.001).

\section{Participants}

From April 2015 to September 2016, 161 youths were invited to participate in the study from the usual inflow of patients at three YMHC and two YAC facilities in the Netherlands. Eligible patients were 13-23 years old, who started outpatient mental health or addiction treatment, were willing to participate in the study and provided written informed consent (if under the age of 18 years also consent from at least one caregiver). We barred patients from the study if they were cognitively incapable of comprehending the questionnaires (clinical judgement), were diagnosed with DSM-IV autism spectrum disorder, or needed inpatient treatment (clinical judgement).
Informed consent was provided by 153 youths and 137 youths $(89.5 \%)$ completed the baseline assessment (see Fig. 1: Consort Flow diagram). After the baseline assessment, six youths were barred from the study because they needed inpatient treatment $(n=5)$ or were diagnosed with autism spectrum disorder $(n=1)$. Four additional youths were excluded because of withdrawn informed consent. The final sample consisted of 127 youths (YMHC: $N=71$; YAC: $N=56)$. From these, 15 youths $(11.8 \%)$ did not participate in the 4-month follow-up assessment (Fig. 1). Fifty-six therapists participated in the study and they treated 1-8 youths each: 23 therapists (41\%) treated one youth; $15(26.8 \%)$ treated two youths; and $18(32.2 \%)$ treated three or more youths.

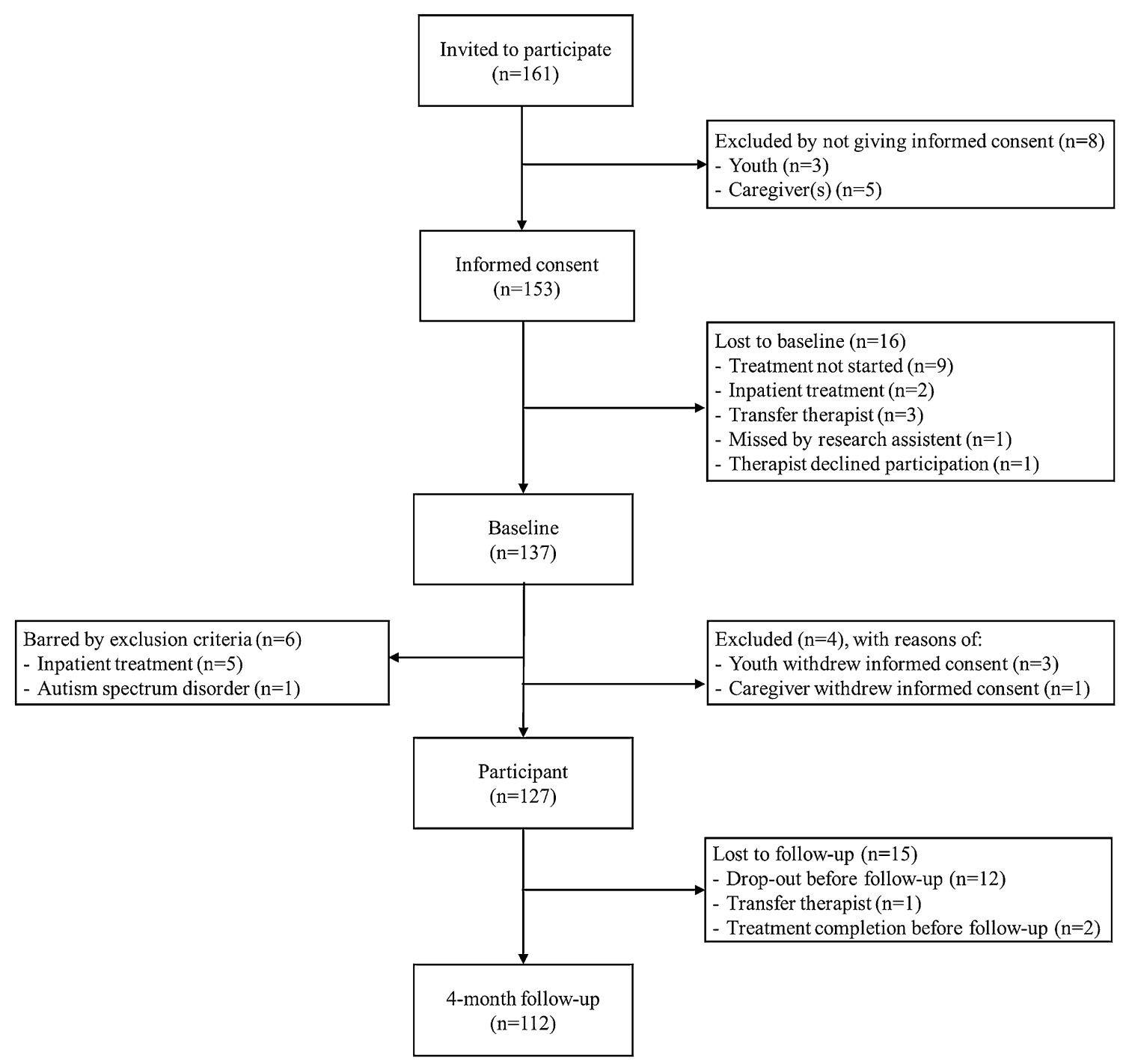

Fig. 1 Flowchart of participants 


\section{Treatment}

Participants were offered individual outpatient cognitive behavioral interventions $(n=93)$, family-based treatment $(n=7)$ or other treatment (i.e. psychomotor therapy and other psychotherapy, $n=24$ ), and type of treatment was not specified for three participants. Median treatment duration was 6 months (interquartile range [IQR]: 4.5-8.0 months) and a median number of 7 sessions was attended (IQR: 4.0-11.0 sessions).

\section{Assessments}

All study assessments, at baseline and 4 months' follow-up, were conducted by trained research assistants. Study assessments included questions about participants' and therapists' demographic background, therapeutic alliance, mental health problems, substance use frequency and diagnosis. Therapeutic alliance was assessed from the perspective of youths and therapists with the Working Alliance Inventory-12 at baseline (WAI-12; [23, 24]; Dutch translation: WAV-12; [25]). The WAI-12 is a 12-item instrument with three subscales: the affective quality of the client-therapist relationship ("Bond"), the degree of agreement on the treatment goals ("Goals"), and the level of task collaboration ("Task"), based on Bordins [26] conceptualization of therapeutic alliance. Youths and therapists were required to rate each item on a 5-point Likert scale ranging from 'never' to 'always'. We used the WAI Total score (range 1-5), as an indication of the overall alliance quality with higher scores indicating better quality of the therapeutic alliance.

To assess youth self-reported mental health problems at baseline and 4 months' follow-up, the Strengths and Difficulties Questionnaire (SDQ; [27]: Dutch translation: [28]) was administered. The SDQ is a commonly applied screening and treatment-****outcome measure with 25 items with a 3-point Likert scale, ranging from 'not true' to 'certainly true'. We used the SDQ Total score (range 0-40), with higher scores indicating more problems. The substance use section of the Measurements in the Addictions for Triage and Evaluation, Youth version (MATE-Y; [29]) was used to collect past month information on the youths' primary substance or behavioral addiction (gaming/gambling) at baseline and 4 months follow-up. Clinical psychiatric diagnosis at baseline was made by the treating therapist, who used the criteria of the Diagnostic and Statistical Manual of Mental Disorders [30].

\section{Primary outcome measure}

We used a prespecified dichotomous outcome measure reflecting a favorable versus unfavorable treatment outcome status at 4-month follow-up as the primary outcome measure for both youths in YMHC and YAC. Youths in YMHC were considered to have a favorable outcome status if their 4-month SDQ total score was lower than 12.5. In the absence of formal Dutch cut-off scores [31], we followed the procedures suggested by Jacobson and Truax [32] and De Beurs et al. [33] to determine the cut-off value of 12.5 as the average of the mean SDQ total score in a Dutch general youth population sample $(\mathrm{M}=9.7, \mathrm{SD}=4.7$; [28] $)$ and the mean baseline SDQ total score in our clinical population $(\mathrm{M}=15.3, \mathrm{SD}=5.4)$. Youths in YAC were considered to have a favorable outcome status if they had used their primary substance or displayed their primary gaming/gambling on less than five days in the 30 days preceding the 4-month follow-up, as recommended in the guidelines for routine outcome monitoring (ROM) in Dutch addiction care (Blanken, et al. 2011, Note from Dutch Expertgroup ROM-Addiction care).

\section{Data-analysis}

Since our alliance data were nested within two levels of clustering we explored the option of using multi-level modeling, but due to insufficient sample size at both levels (level-1: 56 therapists; $M=2.27$ youth per therapist; range 1-8 and level2: five treatment facilities, range 4-11 therapists per treatment facility) it was not possible to estimate effects accurately [34]. In order to address the first study goal, pertaining to the prognostic importance of youth- and therapist-rated alliance for treatment outcome, we conducted two separate multivariate logistic regression analyses including either the youth- or the therapist-rated alliance as independent variable and treatment outcome status (favorable versus unfavorable) as dependent variable. In both regression analyses, we examined the effects of potential confounders: gender, age group ( $\leq 16$ or $\geq 17$ years), treatment setting (YMHC or YAC), cultural background (Dutch or Non-Dutch), education level (low or high), baseline problem status (favorable or unfavorable) on the primary problem domain (mental health status for youth in YMHC; substance use status for youth in YAC), and baseline problem status (favorable or unfavorable) on the concurrent problem domain (substance use status for youth in YMHC; mental health status for youth in YAC). A variable was considered to be a relevant confounder when the youth- or therapist-rated therapeutic alliance regression coefficient changed with $10 \%$ or more after adding the potential confounder into the logistic regression model [35].

In order to address our second study goal, pertaining to the added predictive value for treatment outcome of incorporating the alliance ratings from youths as well as therapists, we conducted a logistic regression analysis in which both youth- and therapist-rated alliance were entered into the model, again with a favorable or unfavorable treatment outcome as dependent variable. A test of improved prediction 
accuracy was conducted by comparing the difference in -2 $\log$ likelihood between the two models, in which we considered a change of $\geq 3.84(\mathrm{df}=1 ; p=0.05)$ as an indication of an improved prediction model.

Finally, we used the median value of the youth-rated and the therapist-rated alliance to distinguish four mutually exclusive subgroups, with alliance rated as (1) "strong" by both youth and therapist, (2) "weak" by the youth and "strong" by the therapist, (3) "strong" by the youth and "weak" by the therapist, and (4) "weak" by both youth and therapist. We then conducted a logistic regression analysis with the subgroup categories as independent variable to predict treatment outcome status, using the first (strongstrong) and the fourth (weak-weak) category as reference category in two separate analyses. To estimate the treatment outcome status of youth with a missing 4-month follow-up assessment, we did not use statistical imputation, but instead asked the treating therapist to provide a 'best estimate' of the youth's outcome status. All statistical analyses were conducted with IBM SPSS Statistics for Windows, version 25.0 (IBM Corp., Armonk, N.Y., USA).

\section{Results}

The baseline characteristics of the study population are summarized in Table 1. Study participants in YMHC (55.9\%) were mainly females (74.6\%), on average 16.8 years old, and

Table 1 Participant characteristics at baseline

\begin{tabular}{|c|c|c|c|}
\hline & $\begin{array}{l}\text { Youth mental health care } \\
(n=71) \% / \text { mean }(\mathrm{sd}) \\
\text { median }\end{array}$ & $\begin{array}{l}\text { Youth addiction care } \\
(n=56) \% / \text { mean }(\mathrm{sd}) \\
\text { median }\end{array}$ & $\begin{array}{l}\text { Total sample } \\
(n=127) \% / \text { mean } \\
(\mathrm{sd}) \text { median }\end{array}$ \\
\hline \multicolumn{4}{|l|}{ Demographic background } \\
\hline Age (13-23) (years) & $16.8(2.1) \mathbf{1 6 . 0}$ & $19.5(2.2) \mathbf{2 0 . 0}$ & $18.0(2.5) \mathbf{1 8 . 0}$ \\
\hline Aged $\leq 16(\%)$ & 53.5 & 16.1 & 37.0 \\
\hline Male $(\%)$ & 25.4 & 83.9 & 51.2 \\
\hline Cultural background Non-Dutch (\%) & 25.4 & 23.2 & 24.4 \\
\hline Education level low (\%) & 64.8 & 58.9 & 62.2 \\
\hline \multicolumn{4}{|l|}{ Substance use } \\
\hline \multicolumn{4}{|l|}{ Primary substance/addictive disorder (\%) } \\
\hline Cannabis use disorder & - & 51.8 & 22.8 \\
\hline Gaming/gambling disorder & - & 17.9 & 7.9 \\
\hline Alcohol use disorder & - & 16.1 & 7.1 \\
\hline Hard drug use disorder & - & 14.3 & 6.3 \\
\hline Days primary substance use/problem behavior past month & $5.4(9.9) \mathbf{0 . 0}$ & $14.3(12.3) \mathbf{1 3 . 0}$ & 9.3 (11.9) 2.0 \\
\hline Problematic substance use past month ( $\geq 5$ days) $(\%)$ & 25.4 & 62.5 & 41.7 \\
\hline \multicolumn{4}{|l|}{ Mental health } \\
\hline \multicolumn{4}{|l|}{ Primary non-addiction disorder (\%) } \\
\hline Mood disorder & 29.6 & - & 16.5 \\
\hline Anxiety disorder & 26.8 & - & 15.0 \\
\hline Behavioral disorder & 22.5 & - & 12.6 \\
\hline Attention Deficit Hyperactivity Disorder (ADHD) & 7.0 & - & 3.9 \\
\hline Other disorder & 14.1 & - & 7.9 \\
\hline Strengths and Difficulties Questionnaire (SDQ score: 0-40) & $15.4(5.4) \mathbf{1 6 . 0}$ & $15.1(5.6) \mathbf{1 5 . 0}$ & $15.3(5.4) \mathbf{1 5 . 0}$ \\
\hline Problematic mental health status (SDQ score $\geq 12.5)(\%)$ & 69.0 & 66.1 & 67.7 \\
\hline \multicolumn{4}{|l|}{ Treatment } \\
\hline \multicolumn{4}{|l|}{ Treatment type $(\%)$} \\
\hline Cognitive behavioral interventions & 58.0 & 96.4 & 75.0 \\
\hline Family-based treatment & 7.2 & 3.6 & 5.6 \\
\hline Other & 34.8 & - & 19.4 \\
\hline Concurrent pharmacological treatment: Yes (\%) & 26.5 & 7.3 & 17.9 \\
\hline \multicolumn{4}{|l|}{ Therapeutic alliance } \\
\hline Youth-rated WAI (1-5) & $3.8(0.7) 3.9$ & $4.0(0.6) 4.1$ & $3.9(0.7) 4.1$ \\
\hline Therapist-rated WAI (1-5) & $3.8(0.5) 4.0$ & $4.0(0.5) 4.1$ & $3.9(0.5) 4.0$ \\
\hline
\end{tabular}


diagnosed with a primary mood $(29.6 \%)$, anxiety $(26.8 \%)$ or behavioral $(22.5 \%)$ disorder. Youth in YAC (44.1\%) were predominantly males (83.9\%), on average 19.5 years old, and diagnosed with a primary cannabis use disorder (51.8\%). Compared with youths in YMHC, youths in YAC were older $(t(125)=-6.95, p<0.001)$, more often male $\left(X^{2}(1)=42.99, p<0.001\right)$ and more often had an unfavorable problem status on the concurrent problem domain $\left(X^{2}(1)=16.80, p<0.001\right)$.

\section{4-month treatment outcome}

The proportion of youths with an unfavorable problem status decreased from $66.1 \%$ at baseline to $56.7 \%$ at 4 months' follow-up (McNemar $X^{2}$ test, $p=0.052$ ).

\section{Baseline therapeutic alliance as predictor for 4-month treatment outcome}

The unadjusted regression coefficient of baseline youth-rated alliance for predicting outcome was $b=1.18(p<0.001$; OR 3.25) (Table 2). From all potential confounders, only 'gender' (highlighted in bold in Table 2) changed the regression coefficient of the association between youth-rated therapeutic alliance and 4-month treatment outcome with more than $10 \%$ (from 1.18 to 1.29 ) and was, therefore, considered to be a relevant confounder. In the final model, the youth-rated therapeutic alliance-outcome association adjusted for gender was $b=1.29(p<0.001$; OR 3.65).

The unadjusted regression coefficient for therapist-rated alliance was $b=1.10(p<0.005$; OR 3.02). Relevant confounders for the association between therapist-rated alliance and 4-month treatment outcome were 'age group' and 'baseline problem status' (highlighted in bold in Table 2). In the final model, the therapist-rated therapeutic alliance-outcome association adjusted for age group and baseline problem status was $b=1.12(p<0.05$; OR 3.07).

\section{Incorporating both alliance perspectives to predict 4-month treatment outcome}

The youths' and therapists' alliance ratings were not interrelated (Spearman's rank correlation $=0.08 ; p=0.38$ ). Concerning the predictive value for treatment outcome of incorporating the alliance ratings of youths and therapists, we found that adding the second perspective significantly improved the logistic regression model, given a $10.75\left(\mathrm{X}^{2}\right.$ $(1, \mathrm{~N}=125)=10.75, p=0.001)$ change in $-2 \log$ likelihood. To illustrate the prognostic significance of incorporating both perspectives in clinical practice, we distinguished four subgroups (Table 3). In the subgroup where both youth and therapist considered the first-session alliance as weak ( $24.8 \%$ of the study sample), the proportion of youths with a favorable 4-month outcome amounted to $22.6 \%$, and in the subgroup where both raters considered the alliance as strong (29.6\%) this proportion amounted to $70.3 \%$. Compared with the subgroup with strong youth- and strong therapist-rated alliance, the odds ratio of a favorable treatment outcome was significantly lower in each of the three other subgroups (Table 3). Conversely, the odds of a favorable outcome in the subgroup with a strong alliance according to both perspectives were more than eight times higher (large effect) than the odds of a favorable outcome in the subgroup with a weak alliance from both perspectives $(\mathrm{OR}=8.10 ; 95 \%$-CI 2.70-24.30, $p<0.001)$.
Table 2 Unadjusted and adjusted associations between therapeutic alliance and 4-month treatment outcome

\begin{tabular}{|c|c|c|c|c|c|c|}
\hline & \multicolumn{3}{|c|}{ Youth-rated therapeutic alliance } & \multicolumn{3}{|c|}{$\begin{array}{l}\text { Therapist-rated therapeutic alli- } \\
\text { ance }\end{array}$} \\
\hline & $\mathrm{b}$ & OR & $95 \%-\mathrm{CI}$ of OR & $\mathrm{b}$ & OR & $95 \%-C I$ of OR \\
\hline Unadjusted model & 1.18 & 3.25 & $1.67-6.30$ & 1.10 & 3.02 & $1.39-6.57$ \\
\hline \multicolumn{7}{|l|}{ Adjusted for } \\
\hline Gender & 1.29 & 3.65 & $1.82-7.29$ & 0.80 & 2.67 & $1.23-5.82$ \\
\hline Treatment setting & 1.15 & 3.14 & $1.62-6.12$ & 1.04 & 2.83 & $1.29-6.21$ \\
\hline Age group & 1.17 & 3.21 & $1.65-6.25$ & 0.99 & 2.70 & $1.25-5.85$ \\
\hline Cultural background & 1.19 & 3.30 & $1.69-6.45$ & 1.10 & 3.01 & $1.38-6.55$ \\
\hline Education & 1.17 & 3.24 & $1.67-6.27$ & 1.12 & 3.07 & $1.40-6.71$ \\
\hline Concurrent problem domain ${ }^{a}$ & 1.18 & 3.27 & $1.68-6.35$ & 1.09 & 2.98 & $1.36-6.52$ \\
\hline Baseline problem status & 1.12 & 3.06 & $1.46-6.41$ & 1.20 & 3.32 & $1.38-7.98$ \\
\hline Adjusted final model & $1.29^{\mathrm{b}}$ & 3.65 & $1.82-7.29$ & $1.12^{\mathrm{c}}$ & 3.07 & $1.24-7.61$ \\
\hline
\end{tabular}

Relevant confounders ( $\geq 10 \%$ change in $b)$ in bold

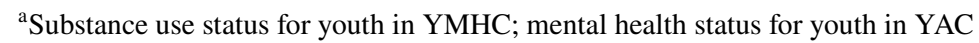

${ }^{\mathrm{b}}$ Adjusted for gender

${ }^{\mathrm{c}}$ Adjusted for age group and baseline problem status 
Table 3 Incorporating alliance ratings of youths and therapists to predict 4-month treatment outcome

\begin{tabular}{|c|c|c|c|c|c|c|c|}
\hline & Ratin youth & Rating therapist ${ }^{\mathrm{a}}$ & $n(\%)$ & $\begin{array}{l}\% \text { favorable } 4 \text {-month } \\
\text { treatment outcome }\end{array}$ & OR & $95 \%$-CI & $p$ \\
\hline \multirow[t]{4}{*}{ Therapeutic alliance } & Weak & Weak & $31(24.8)$ & 22.6 & 0.12 & $0.04-0.37$ & $<.001$ \\
\hline & Strong & Weak & $25(20.0)$ & 32.0 & 0.20 & $0.07-0.60$ & $<.05$ \\
\hline & Weak & Strong & $32(25.6)$ & 40.6 & 0.29 & $0.11-0.79$ & $<.05$ \\
\hline & Strong $^{\mathrm{b}}$ & Strong $^{\mathrm{b}}$ & $37(29.6)$ & 70.3 & - & - & - \\
\hline
\end{tabular}

${ }^{\mathrm{a}} n=125$; two therapist-rated therapeutic alliance missings

${ }^{\mathrm{b}}$ Reference group

\section{Discussion}

The aims of our study were to investigate the prognostic importance of first-session youth- and therapist-rated therapeutic alliance and the added predictive value of incorporating these alliance ratings for outcome of youth in YMHC and YAC. Youth- and therapist-rated alliance were not related to each other and each showed a medium association with treatment outcome in both YMHC and YAC. Our findings suggest that incorporating the alliance perspectives of both youths and therapists provides substantially stronger predictive value for treatment outcome than using one perspective only. To illustrate the relevance of this finding for clinical practice, we distinguished four subgroups based on the alliance ratings from both perspectives, and found these subgroups to differ considerably in treatment outcome, with the lowest proportion of favorable outcome in the subgroup where both youth and therapist considered the alliance as weak $(23 \%)$, and the highest proportion in the subgroup where both raters considered the alliance as strong (70\%).

Overall, the medium effect sizes of the alliance-outcome associations in our study are in line with the effect sizes found in previous systematic reviews on therapeutic alliance in adolescents [7, 8] and adults [6]. However, we found similar alliance-outcome associations for SUD as for non-SUD mental health disorders, whereas Fluckiger et al. [6] and Karver et al. [7] reported lower associations for substance use disorders. Our finding that youth- and therapist-rated alliance were not interrelated matches previous research showing nonsignificant correlations between both at the start of treatment $[11,18]$, and these findings suggest that youth and therapist alliance perspectives differ substantially. Most previous studies that have investigated therapeutic alliance from more than one perspective focused on the issue which of these perspectives showed the strongest association with treatment outcome. To the best of our knowledge, our study is the first in which the alliance evaluations from both the therapist and youth were incorporated to maximize their predictive value for treatment outcome.

Our findings further suggest that it may be worthwhile to start evaluating the therapeutic relationship when initiating therapy. For therapists offering psychosocial services to youth, paying attention to first-session therapeutic alliance may be particularly relevant since youths generally enter treatment on account of parental and environmental concerns and often show distrust of adult authority and have a strong desire for autonomy [36, 37]. Therapists can employ different alliance building techniques to improve therapeutic alliance [38, 39]. Furthermore, regular measurement of the therapeutic alliance throughout the course of treatment may be part of a broader approach, such as Feedback Informed Treatment (FIT) [40] and can be applied in different forms and through different feedback systems [41]. A recent metaanalysis on the application of FIT in psychological services with youth showed an overall beneficial effect in the small range [42]. Although promising, more research on the application of FIT in youth mental health care is warranted.

Our study has several limitations that should be mentioned. First, the use of multi-level modeling was precluded because of the majority of therapists with few $(<2)$ participating youths; the minority of therapists with a higher number (3-8) of participating youths; and the limited therapists per treatment facility. Nevertheless, we found no difference in alliance ratings between therapists with few participating youths versus therapists with more participating youths; in addition, we found that treatment setting was not a relevant confounder in this study. Second, our findings regarding the association between therapeutic alliance and treatment outcome are merely correlational in nature. To allow stronger causal inferences, Cuijpers and colleagues [43] argue that there should be a temporal association, a dose-response relationship, exclusion of alternative potential mediators, preferably experimental manipulation, and a plausible theoretical explanatory framework. From these, we did take the temporal association into account, as well as possible confounders and moderators of the association, and we measured the alliance as early as possible in the therapeutic process to minimize potential confound due to early symptom improvement. In addition, we believe that the theoretical framework pertaining to the role of common factors like alliance in therapy is not weaker than that of, e.g., specific factors in cognitive behavioral therapy. Third, the focus on month- 4 
treatment outcome and the lack of long-term follow-up data does not allow for conclusions about the relevance of initial alliance ratings for predicting long-term outcome. Nevertheless, there is evidence that most symptom improvement occurs during the first phase of treatment [20-22, 44].

To conclude, this study supports the importance of including both perspectives on therapeutic alliance for treatment outcome in youth and suggests that incorporating both youths' and therapists' alliance perspectives has more prognostic value than considering only one perspective.

Acknowledgements The authors would like to thank all youths and therapists for their participation and all students for their help with the data collection. This research was supported by The Netherlands Organization for Health Research and Development.

\section{Compliance with ethical standards}

Conflict of interest: The authors declare no conflict of interest.

Open Access This article is licensed under a Creative Commons Attribution 4.0 International License, which permits use, sharing, adaptation, distribution and reproduction in any medium or format, as long as you give appropriate credit to the original author(s) and the source, provide a link to the Creative Commons licence, and indicate if changes were made. The images or other third party material in this article are included in the article's Creative Commons licence, unless indicated otherwise in a credit line to the material. If material is not included in the article's Creative Commons licence and your intended use is not permitted by statutory regulation or exceeds the permitted use, you will need to obtain permission directly from the copyright holder. To view a copy of this licence, visit http://creativecommons.org/licenses/by/4.0/.

\section{References}

1. Merikangas KR et al (2010) Lifetime prevalence of mental disorders in U.S. adolescents: results from the National Comorbidity Survey Replication-Adolescent Supplement (NCS-A). J Am Acad Child Adolesc Psychiatry 49(10):980-989

2. Ormel J et al (2015) Mental health in Dutch adolescents: a TRAILS report on prevalence, severity, age of onset, continuity and co-morbidity of DSM disorders. Psychol Med 45(2):345-360

3. Weisz JR et al (2017) What five decades of research tells us about the effects of youth psychological therapy: a multilevel metaanalysis and implications for science and practice. Am Psychol 72(2):79-117

4. Bender $\mathrm{K}$ et al (2011) A meta-analysis of interventions to reduce adolescent cannabis use. Res Soc Work Pract 21(2):153-164

5. Tanner-Smith EE, Wilson SJ, Lipsey MW (2013) The comparative effectiveness of outpatient treatment for adolescent substance abuse: a meta-analysis. J Subst Abuse Treat 44(2):145-158

6. Flückiger $\mathrm{C}$ et al (2018) The alliance in adult psychotherapy: A meta-analytic synthesis. Psychotherapy (Chicago III) 55(4):316-340

7. Karver $\mathrm{M}$ et al (2018) Meta-analysis of the prospective relation between alliance and outcome in child and adolescent psychotherapy. Psychotherapy (Chicago III) 55(4):341-355

8. Murphy R, Hutton P (2018) Practitioner Review: Therapist variability, patient-reported therapeutic alliance, and clinical outcomes in adolescents undergoing mental health treatment - a systematic review and meta-analysis. J Child Psychol Psychiatry 59(1):5-19

9. Elvins R, Green J (2008) The conceptualization and measurement of therapeutic alliance: an empirical review. Clin Psychol Rev 28(7):1167-1187

10. Lamers A, Vermeiren RR (2015) Assessment of the therapeutic alliance of youth and parents with team members in youth residential psychiatry. Clin Child Psychol Psychiatry 20(4):640-656

11. Fjermestad KW et al (2016) Therapist-youth agreement on alliance change predicts long-term outcome in CBT for anxiety disorders. J Child Psychol Psychiatry 57(5):625-632

12. Bickman L et al (2012) The relationship between change in therapeutic alliance ratings and improvement in youth symptom severity: whose ratings matter the most? Adm Policy Ment Health 39(1-2):78-89

13. Darchuk AJ (2007) The role of the therapeutic alliance and its relationship to treatment outcome and client motivation in an adolescent substance abuse treatment setting. Ohio University, Ohio

14. Diamond $\mathrm{G}$ et al (2006) Early therapeutic alliance as a predictor of treatment outcome for adolescent cannabis users in outpatient treatment. Am J Addict 15(Suppl 1):26-33

15. Handwerk ML et al (2008) The Role of Therapeutic Alliance in Therapy Outcomes for Youth in Residential Care. Resid Treat Child Youth 25(2):145-165

16. Ormhaug SM, Shirk S, Wentzel-Larsen T (2015) Therapist and client perspectives on the alliance in the treatment of traumatized adolescents. Eur J Psychotraumatol 6:27705

17. Shirk $S$ et al (2008) Alliance and outcome in cognitive-behavioral therapy for adolescent depression. J Clin Child Adolesc Psychol 37(3):631-639

18. Duppong Hurley K et al (2015) Examining change in therapeutic alliance to predict youth mental health outcomes. J Emot Behav Disord 23(2):90-100

19. Eltz MJ, Shirk SR, Sarlin N (1995) Alliance formation and treatment outcome among maltreated adolescents. Child Abuse Negl 19(4):419-431

20. Baldwin SA et al (2009) Rates of change in naturalistic psychotherapy: contrasting dose-effect and good-enough level models of change. J Consult Clin Psychol 77(2):203-211

21. Rubel J, Lutz W, Schulte D (2015) Patterns of change in different phases of outpatient psychotherapy: a stage-sequential pattern analysis of change in session reports. Clin Psychol Psychother 22(1):1-14

22. Warren JS et al (2010) Youth psychotherapy change trajectories and outcomes in usual care: Community mental health versus managed care settings. J Consult Clin Psychol 78(2):144-155

23. Horvath AO, Greenberg LS (1986) The development of the Working Alliance Inventory. In: Greenberg LS (ed) The psychotherapeutic process: A research handbook. Guilford Press, New York, pp 529-556

24. Horvath AO, Greenberg LS (1989) Development and validation of the Working Alliance Inventory. J Couns Psychol 36(2):223-233

25. Vertommen H, Vervaeke GAC (1990) Werkalliantievragenlijst (wav). Vertaling voor experimenteel gebruik van de WAI (Horvart \& Greenberg, 1986). Departement Psychologie KU Leuven

26. Bordin ES (1979) The generalizability of the psychoanalytic concept of the working alliance. Psychother Theory Res Pract 16(3):252

27. Goodman R (1997) The strengths and difficulties questionnaire: a research note. J Child Psychol Psychiatry 38(5):581-586

28. van Widenfelt BM et al (2003) Dutch version of the strengths and difficulties questionnaire (SDQ). Eur Child Adolesc Psychiatry 12(6):281-289

29. Schippers GM, Broekman TG (2013) Measurements in the addictions for triage and evaluation (MATE): an instrument based on 
the World Health Organization family of international classifications. Addiction 105(5):862-871

30. American Psychiatric Association (2000) Diagnostic and statistical manual of mental disorders. 4th text rev ed. Washington DC

31. Vugteveen J et al (2018) Psychometric properties of the Dutch strengths and difficulties questionnaire (SDQ) in adolescent community and clinical populations. Assessment. https://doi. org/10.1177/1073191118804082

32. Jacobson NS, Truax P (1991) Clinical significance: a statistical approach to defining meaningful change in psychotherapy research. J Consult Clin Psychol 59(1):12

33. de Beurs E et al (2015) Denoting treatment outcome in child and adolescent psychiatry: a comparison of continuous and categorical outcomes. Eur Child Adolesc Psychiatry 24(5):553-563

34. Schoeneberger JA (2015) The Impact of Sample Size and Other Factors When Estimating Multilevel Logistic Models. J Experim Educat 84(2):373-397

35. Twisk J (2016) Inleiding in de toegepaste biostatistiek. 4th ed. Bohn stafleu van loghum, Houten

36. Block AM, Greeno CG (2011) Examining outpatient treatment dropout in adolescents: A literature review. Child Adolesc Soc Work J 28(5):393-420

37. De Haan AM et al (2013) A meta-analytic review on treatment dropout in child and adolescent outpatient mental health care. Clin Psychol Rev 33(5):698-711
38. Ackerman SJ, Hilsenroth MJ (2003) A review of therapist characteristics and techniques positively impacting the therapeutic alliance. Clin Psychol Rev 23(1):1-33

39. Diamond $\mathrm{G}$ et al (1999) Alliance-building interventions with adolescents in family therapy: a process study. Psychotherapy 36(4):355

40. Bertolino B, Miller SD (2012) ICCE Manuals on feedback informed treatment (FIT)

41. Lambert MJ, Whipple JL, Kleinstauber M (2018) Collecting and delivering progress feedback: a meta-analysis of routine outcome monitoring. Psychotherapy (Chicago III.) 55(4):520-537

42. Tam HE, Ronan K (2017) The application of a feedback-informed approach in psychological service with youth: Systematic review and meta-analysis. Clin Psychol Rev 55:41-55

43. Cuijpers P, Reijnders M, Huibers MJH (2018) The role of common factors in psychotherapy outcomes. Ann Rev Clin Psychol 15:207-231https://doi.org/10.1146/annurev-clinpsy-05071 8-095424

44. Lambert MJ (2005) Emerging methods for providing clinicians with timely feedback on treatment effectiveness: An introduction. J Clin Psychol 61(2):141-144 